Research Paper

\title{
Increased Expression of LAMTOR5 Predicts Poor Prognosis and Is Associated with Lymph Node Metastasis of Head and Neck Squamous Cell Carcinoma
}

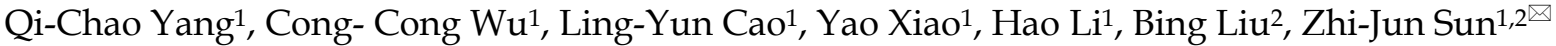 \\ 1. The State Key Laboratory Breeding Base of Basic Science of Stomatology (Hubei-MOST) \& Key Laboratory of Oral Biomedicine Ministry of Education, \\ School and Hospital of Stomatology, Wuhan University, Wuhan, China \\ 2. Department of Oral and Maxillofacial Head Neck Oncology, School \& Hospital of Stomatology, Wuhan University, Wuhan, China
}

$\square$ Corresponding author: Zhi-Jun Sun, M.D., Professor. Address: Department of Oral Maxillofacial- Head Neck Oncology, School and Hospital of Stomatology, Wuhan University, 237 Luoyu Road, Wuhan, Hubei Province, China.430079; Fax: +86 27 8787 3260; E-mail: sunzj@whu.edu.cn

(c) Ivyspring International Publisher. This is an open access article distributed under the terms of the Creative Commons Attribution (CC BY-NC) license (https:// creativecommons.org/licenses/by-nc/4.0/). See http://ivyspring.com/terms for full terms and conditions.

Received: 2019.01.22; Accepted: 2019.05.03; Published: 2019.06.02

\begin{abstract}
Late endosomal/lysosomal adaptor and MAPK and mTOR activator 5 (LAMTOR5) is a novel oncoprotein associated with several human malignancies, but its clinical role in head and neck squamous cell carcinoma (HNSCC) remains unclear. The present study aims to investigate the clinical and pathological significance of LAMTOR5 in HNSCC. We utilized immunohistochemical staining of human tissue microarrays (210 primary HNSCC, 42 normal oral mucosae, 69 oral epithelial dysplasia, and 68 metastasis lymph nodes) to explore the clinical and pathological significance of LAMTOR5 in HNSCC. Additionally, expression level of LAMTOR5 in immunoreactivity of Pten conditional knock out (Pten cKO) mice HNSCC was also assessed. We found LAMTOR5 was overexpressed in human and Pten cKO mice HNSCC, and its expression was significantly associated with patients' overall survival, lymph node metastasis and lymph node grade. Furthermore, LAMTOR5 expression was significantly correlated with the expression of p-AktSer473, p-S6Ser235/236, immune checkpoints (PD-LI, Galectin 9, VISTA and B7-H4) and macrophage markers (CD68 and CD163). In Pten cKO mice HNSCC, it was also significantly correlated with VISTA and F4/80. Consequently, we consider that high expression of LAMTOR5 might be a poor prognostic indicator and correlated with the immunosuppression of tumor microenvironment.
\end{abstract}

Key words: LAMTOR5, head and neck squamous cell carcinoma, prognosis, immunohistochemistry, knockout mice

\section{Introduction}

Head and neck squamous cell carcinoma (HNSCC) is the seventh most common cancer globally, accounting for more than $95 \%$ of head and neck cancers [1]. With an annual new cases of more than 4,00,000 worldwide, two-thirds of the HNSCC cases are reported from developing countries, and only $40-50 \%$ of the patients can reach five-year overall survival $[2,3]$. Unfortunately, the overall survival of patients with HNSCC has not been remarkably improved over the past 30 years, despite advances in traditional treatment for HNSCC, including surgery, radiotherapy and chemotherapy $[4,5]$.

Late endosomal/lysosomal adaptor and MAPK and mTOR activator 5 (LAMTOR5), which is also called mammalian hepatitis B X-interacting protein (HBXIP), was originally identified as an interaction protein with hepatitis B viral protein (HBx) [6]. Abnormal expression of LAMTOR5 causes excessive centrosome production and multipolar mitotic spindles, resulting in chromosome segregation defects and genetic instability, which is actually indicative of emerging malignancy $[7,8]$. Acting as an oncoprotein in promoting cell growth, proliferation, migration and angiogenesis, LAMTOR5 is overexpressed in several malignancies, including esophageal squamous cell carcinoma, non-small-cell lung cancer, liver cancer, ovarian cancer, cervical cancer and breast cancer [9-14]. Recent studies revealed that, in breast cancer 
and liver hepatocellular carcinoma, LAMTOR5 was closely associated with the activation of the PI3K/Akt signaling pathway $[15,16]$. It has been reported that abnormal activation of the PI3K/Akt/mTOR pathway is associated with the development of HNSCC [17]. Additionally, the expression level of LAMTOR5 is found to be correlated with various clinical features and survival outcomes in both esophageal squamous cell carcinoma and ovarian cancer [9, 10]. However, studies pertaining to expression and the prognostic significance of LAMTOR5 in HNSCC have not yet been reported.

Today, immunotherapy is a new and promising approach for several cancers including head and neck cancer [18]. With downregulation of antigen-processing and presentation, secretion of immunosuppressive cytokines, and recruitment of tumor-associated macrophages (TAMs), immunosuppression may play a key role in HNSCC and is the basis of cancer immunotherapy [19]. Recent studies suggest that inhibitory immune checkpoints play important roles in tumor immune evasion and immunosuppression [20]. Immunotherapy targeting immune checkpoint is a promising avenue for the treatment of HNSCC [21]. In this study, the correlation of LAMTOR5 expression with immune related molecules in human HNSCC tissue microarrays were also examined.

\section{Materials and Methods}

\section{Ethical statement}

Our study has been approved by Institutional Medical Ethics Committee of School and Hospital of Stomatology, Wuhan University in accordance with the Institutional Guidelines (2014LUNSHENZI06). This research conforms with The Code of Ethics of the World Medical Association [22].

\section{Patients, tumor samples and human HNSCC tissue microarrays}

With pathological confirmation at the Hospital of Stomatology of Wuhan University, all patients involved in this study were diagnosed with HNSCC. Before the patients underwent surgery, we have obtained their written informed consent. We classified the patients' HNSCC into different clinical stages and determined the histological grades according to the scheme of the World Health Organization guidelines and the Union for International Cancer Control ( $8^{\text {th }}$ edition) [23]. The clinical follow-up was continuously ongoing until death of the patients or the end of the study. We collected primary HNSCC tissues from each patient and constructed with $1.5-\mathrm{mm}$ core and build up paraffin-embedded tissue microarrays.
Validate cohort using custom made human HNSCC tissue microarrays T12-412 TMA2, T15-411 and T17-790. There are 210 confirmed cases of primary HNSCC (exclude 25 recurrent tumor cases of HNSCC, 15 cases with pre-operation radiotherapy treatment, 20 cases with pre-operation inductive TPF chemotherapy and 10 cases with pre-surgical radiotherapy in the same tissue microarray), 42 normal oral mucosae, 69 oral epithelial dysplasia and 68 metastatic lymph nodes. In addition, clinical features, together with overall survival, can be found in all cases.

\section{Pten cKO Mice HNSCC tissues}

Tissue-specific and time-inducible Pten conditional knock out (Pten cKO, Pten ${ }^{\text {flox/flox; }}$ K14-CreERtam, $\mathrm{n}=5$ ) mice HNSCC tissues were gifted by Dr. Ashok B. Kulkarni in National Institute of Dental and Craniofacial Research.

\section{Immunohistochemistry}

Serial cut 4- $\mu m$ paraffin-embedded sections were deparaffinized and rehydrated. The antigen retrieval was performed in $0.01 \mathrm{M}$ citric acid buffer solution $(\mathrm{pH}=6.0)$. To quench endogenous peroxidase activity and block non-specific binding, 3\% hydrogen superoxide and 10\% normal goat serum were subsequently used. The sections were incubated with monoclonal anti-human LAMTOR5 (1:800, Cell Signaling Technology), p-Akt ${ }^{\mathrm{Ser} 473}$ (1:50, Cell Signaling Technology), p-S6Ser235/236 (1:400, Cell Signaling Technology), programmed death ligand 1 (PD-L1) (1:100, Cell Signaling Technology), Galectin 9 (1:1000, Cell Signaling Technology), V-domain suppressor of $\mathrm{T}$ cell activation (VISTA) (1:400, Cell Signaling Technology), B7-homolog 4 (B7-H4) (1:800, Cell Signaling Technology), CD68 (1:50, Zymed) and CD163 (1:50, CWBiotech) or isotype-matched IgG controls at $4{ }^{\circ} \mathrm{C}$ overnight. A secondary biotinylated IgG antibody solution and an avidinbiotin-peroxidase reagent was then added to the sections, and 3,3'-diaminobenzidine tetrachloride was used for colorization. Finally, the slides were counterstained with hematoxylin.

\section{Scoring system, hierarchical clustering and data visualization}

All the sections were scanned by using an Aperio Image Scope CS2 scanner (CA, USA) with background substrate for each section, and they are quantified using Aperio Quantification software (Version 9.1) for nuclear, membrane or pixel quantification [24]. For scanning and quantification, we selected an area of interest in the cancerous or the epithelial area. Then, the formula ( $1 \times$ the percentage of weakly positive staining $)+(2 \times$ the percentage of 
moderately positive staining $)+(3 \times$ the percentage of strongly positive staining) was applied to count histoscore of membrane and nuclear staining. Histoscore of pixel quantification was calculated as total intensity/total cell number. In line with the standard controls (provided by Aperio), we fixed the threshold used for scanning of different positive cells [25]. The scaled values of expression scores were subsequently converted in Microsoft Excel. Then, we utilized Cluster 3.0 with average linkage, which is based on Person's correlation coefficient, to complete the hierarchical analysis [25]. Java TreeView (Version 1.0.5) were used to visualize the results [26].

\section{Statistical analysis}

All data analyses in this study were conducted with the GraphPad Prism version 7.0 (GraphPad Software Inc., La Jolla, CA) statistical package. Multiple group comparisons were completed with the one-way analysis of variance method, and two-group comparisons were analyzed with the unpaired $t$ test method. For the purpose of generating survival curves and assessing the significance of observed differences, we separated the patients into either the high expression group or the low expression group by using the median expression value or the best cut-off [27] and then applied the Kaplan-Meier log-rank test, respectively. Quantified results were expressed as the mean \pm SEM. When $p<0.05$, the result was considered statistically significant. To build a multivariate Cox proportional hazard model, we applied IBM SPSS statistics 24.0. After confirming a Gaussian distribution of the sample, we used the two-tailed Pearson's statistics to analyze the correlation between expression of LAMTOR5 and p-AktSer473, p-S6 Ser235/236, PD-L1, Galectin 9, VISTA, B7-H4, CD68 and CD163.

\section{Results}

\section{LAMTOR5 was overexpressed in human HNSCC and significantly correlated with patients' overall survival}

We performed immunohistochemistry on human HNSCC tissue microarrays and then analyzed the protein expression of LAMTOR5 in HNSCC. Mainly expressed in the cell cytoplasm and membrane in HNSCC, the expression of LAMTOR5 was found to be significantly higher in HNSCC $(\mathrm{n}=$ 210) than in normal oral mucosa (Fig. 1 . A and $B, n=$ $42, p=0.0414$ ) and dysplasia tissues (Fig. 1. B, $\mathrm{n}=69, p$ $=0.0031$, while the difference between dysplasia tissues and normal oral mucosa was not significant (Fig. 1. B, $p>0.05$ ). Furthermore, we used Kaplan-Meier survival analysis to evaluate the influence of different LAMTOR5 expression levels on the prognosis of HNSCC. The median cut-off of LAMTOR5 expression (histoscore $=83.77$ ) and the best cut-off [27] (histoscore $=34.87$ ) were used respectively. As shown in Fig. 1. C and D, log-rank analysis indicated that the HNSCC patients with higher LAMTOR5 expression had a significantly poorer overall survival compared with the HNSCC patients with lower LAMTOR5 expression $(p=$ 0.0266). Moreover, the difference was much more significant when using the best cut-off [27] $(p=$ $0.0004)$. We utilized the multivariate Cox proportional hazard model with covariates including gender, age, smoking and drinking habits, pathological grade, tumor size, lymph node stage and the LAMTOR5 expression to determine parameters with prognostic significance in HNSCC through multivariate analysis. The results showed that high LAMTOR5 expression, together with pathological grade and tumor size, was significantly associated with poor survival of HNSCC patients (Table 1).

Table 1. Multivariate analysis for overall survival in primary HNSCC patients

\begin{tabular}{lll}
\hline Parameters & HR $(95 \% \mathrm{CI})$ & $P$ value \\
\hline Gender & $0.912(0.398-2.086)$ & 0.827 \\
Age & $1.681(0.893-3.163)$ & 0.107 \\
Smoking & $0.946(0.433-2.066)$ & 0.890 \\
Drinking & $1.058(0.524-2.135)$ & 0.875 \\
Pathological grade & & \\
II vs. I & $24.255(3.230-182.120)$ & $0.002^{*}$ \\
III vs. I & $17.168(2.154-136.858)$ & $0.007^{*}$ \\
Tumor size & & \\
T2 vs. T1 & $1.432(0.561-3.658)$ & 0.453 \\
T3 vs. T1 & $2.382(0.859-6.603)$ & 0.095 \\
T4 vs. T1 & $3.290(1.075-10.069)$ & $0.037^{*}$ \\
Node stage & & \\
N1 vs. N0 & $0.511(0.229-1.141)$ & 0.101 \\
N2 vs. N0 & $1.415(0.684-2.925)$ & 0.349 \\
LAMTOR5 expression & $4.794(1.642-13.992)$ & $0.004^{*}$ \\
\hline Cox proportional hazards regression model & \\
HR hazard ration, 95\% CI 95\% confidence interval & \\
* $p<0.05$ & &
\end{tabular}

\section{Clinical and pathological significance of LAMTOR5 expression in human HNSCC}

The expression of LAMTOR5 in metastatic lymph nodes was significantly higher than in primary HNSCC tissues and was also significantly correlated with lymph node grade. One-way ANOVA and Student's $t$ test were used to evaluate the clinical and pathological significance of LAMTOR5 expression in human HNSCC. Comparison between metastatic lymph nodes and primary HNSCC tissues revealed a significant difference of LAMTOR5 expression, with higher expression in metastatic lymph nodes than that of primary HNSCC tissues (HNSCC vs. LN, $p=$ 0.0417, Fig. 2. A and B). Furthermore, the results indicated that the expression of LAMTOR5 was significantly correlated with lymph node stage (N0 vs. 
$\mathrm{N} 1+\mathrm{N} 2, p=0.0173$, Fig. 2. C) but not with pathological grade (I, II and III, Fig. 2. D, $p>0.05$ ) or tumor size (T1, T2, T3 and T4, $p>0.05$, Fig. 2. E). No significant difference was found between primary HNSCC and recurrent HNSCC (HNSCC vs. recurrence, $p>0.05$, Fig. 2. F), primary HNSCC and presurgical inductive chemotherapy (HNSCC vs. TPF, $p>0.05$, Fig. 2. G) or primary HNSCC and pre-surgical radiotherapy (HNSCC vs. RT, $p>0.05$,
Supplementary Fig. A). Moreover, our study showed that neither human papillomavirus (HPV) infection status nor environmental exposure to tobacco products or alcohol were associated with a significant difference in LAMTOR5 expression (smoking vs. non-smoking, $p>0.05$, Supplementary Fig. B; drinking vs. nondrinking, $p>0.05$, Supplementary Fig. C; $\mathrm{HPV}^{+}$vs. HPV-, $p>0.05$, Supplementary Fig. D).

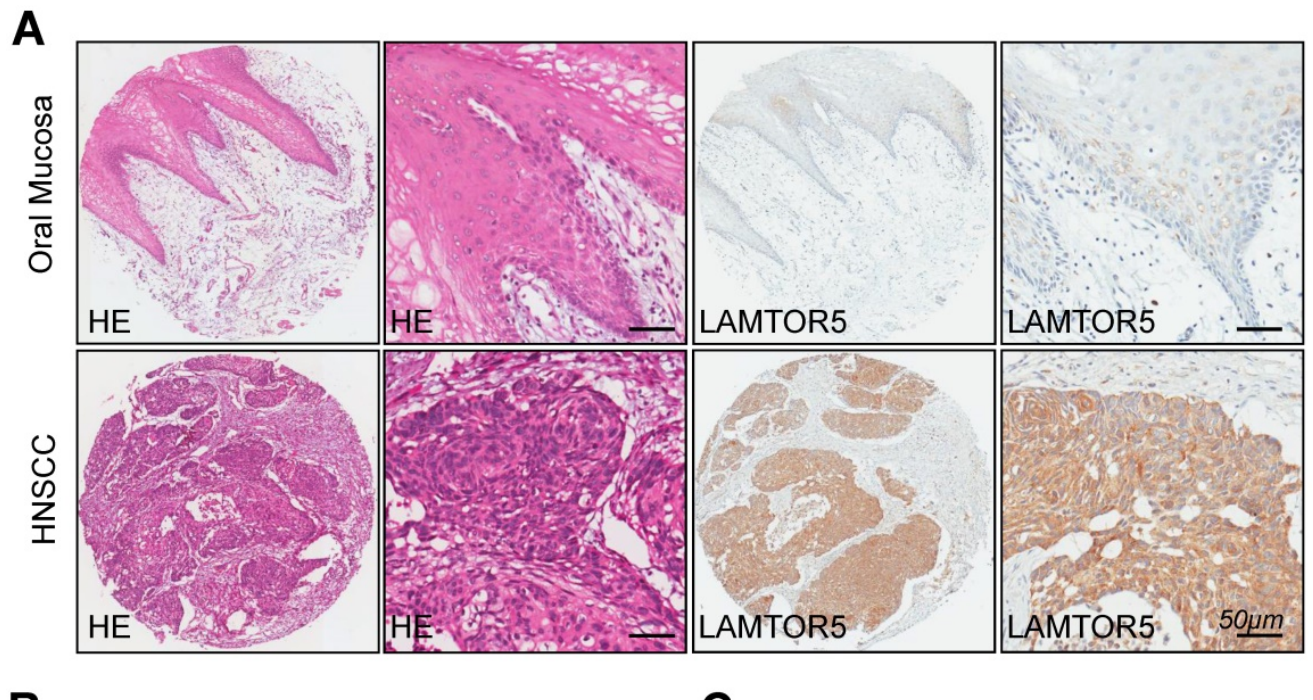

B
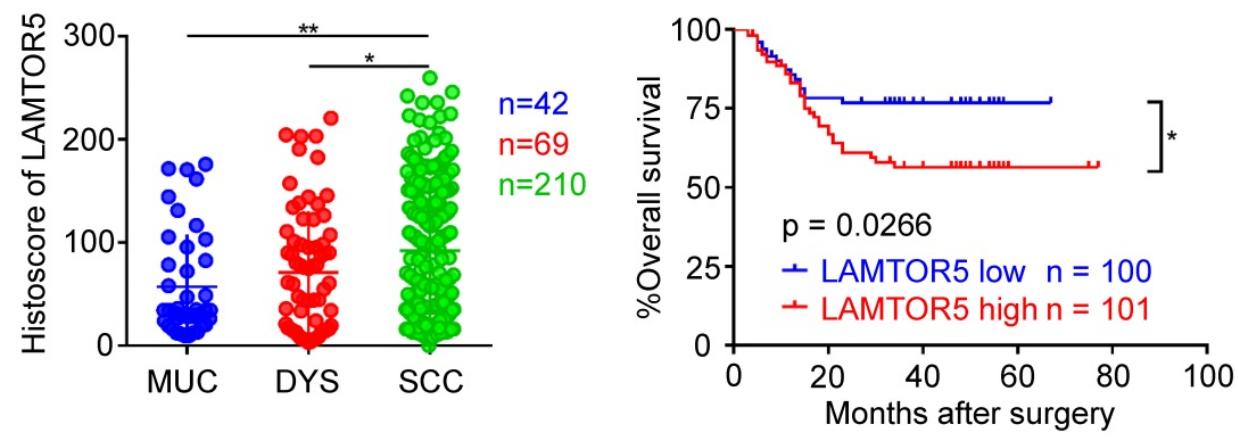

D

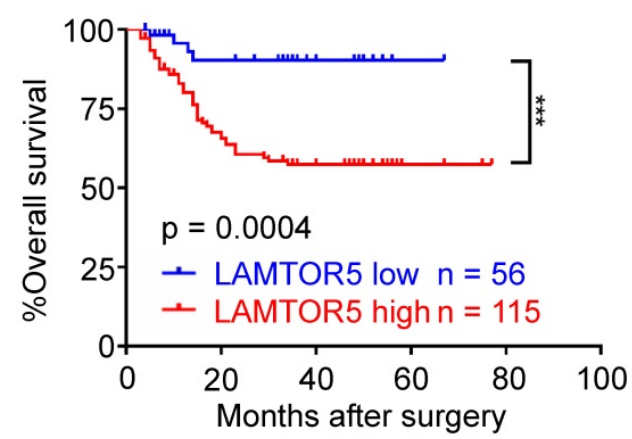

Fig. 1. Overexpression of LAMTOR5 in primary HNSCC. (A) Representative hematoxylin-eosin (HE, left) and immunohistochemical staining (right) of LAMTOR5 in normal mucosa and in primary HNSCC tissue $($ Scale bar $=50 \mu \mathrm{m})$. (B) Quantification of immunohistochemical histoscore of LAMTOR5 among oral mucosa $($ MUC, $\mathrm{n}=42)$, dysplasia (DYS, $n=69$ ) and primary HNSCC $(S C C, n=210)$ (presented as the mean \pm SEM, ordinary one-way ANOVA; MUC vs. SCC, $p=0.0031, * *, p<0.01 ;$ DYS vs. SCC, $p=0.0414, *<<0.05$ ). (C) Kaplan-Meier curve showing that HNSCC patients with low LAMTOR5 expression $(n=100)$ on average survive longer than those with high LAMTOR5 expression $(n=101)$ (we differentiate the expression level by using the mid-value); log-rank analysis revealed the difference was significant $(p=0.0266, *, p<0.05)$. (D) By applying the best cut-off to distinguish the patients with low expression of LAMTOR5 $(n=56)$ from the patients with high expression of LAMTOR5 ( $=115)$, the difference was much more obvious and significant $(p=0.0004 ; * * *, p<0.001)$. 
A

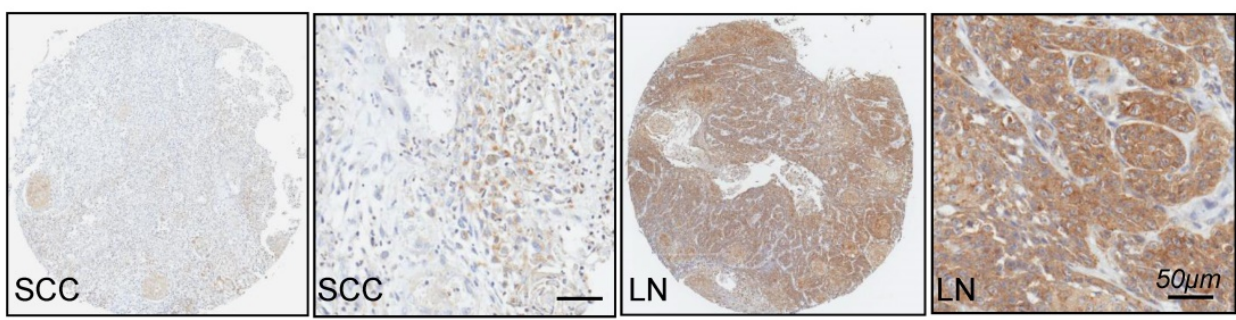

B

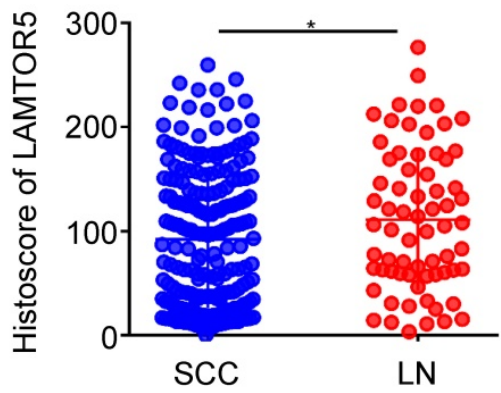

C

D

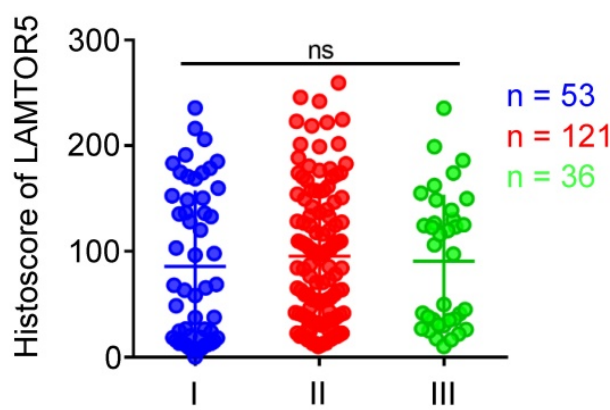

$\mathbf{F}$

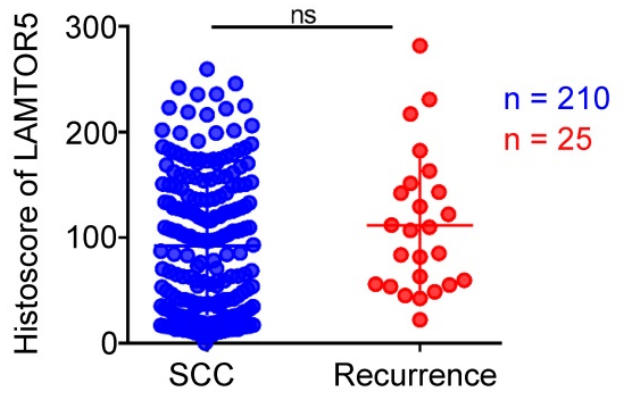

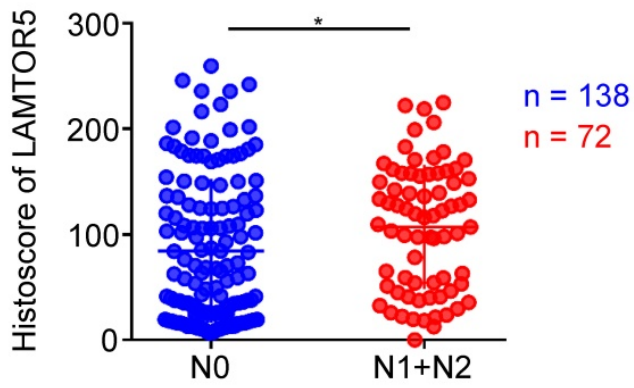

E

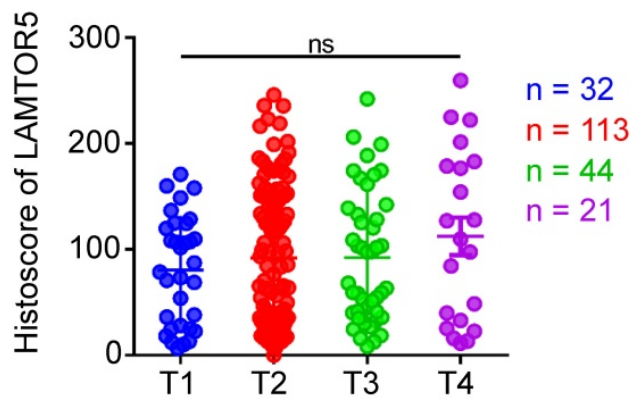

$\mathbf{G}_{2}$

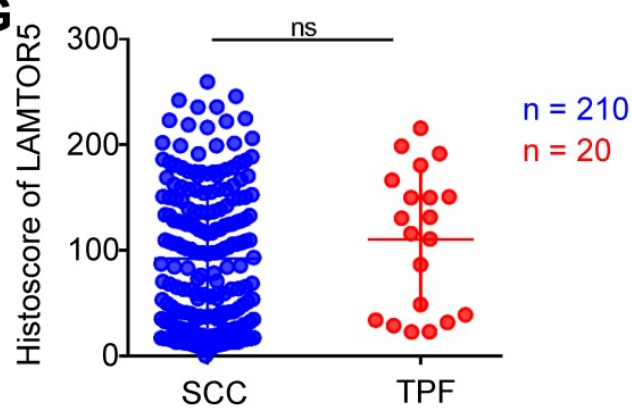

Fig. 2. Increased expression of LAMTOR5 in HNSCC with lymph node metastases. (A) Representative immunohistochemical staining of LAMTOR5 in primary HNSCC tissue (SCC, left) and metastatic lymph node. (LN, right; Scale bar $=50 \mu \mathrm{m}$ ). (B) Quantitative comparison of immunohistochemical histoscore of LAMTOR5 between primary HNSCC (SCC, $\mathrm{n}=210)$ and metastatic lymph nodes $(\mathrm{LN}, \mathrm{n}=68$, unpaired $t$ test, $p=0.0417, *, p<0.05)$. (C) The expression of LAMTOR5 was significantly related to lymph node status of primary HNSCC (N0: $\mathrm{n}=138 ; \mathrm{N} 1+\mathrm{N} 2: \mathrm{n}=72$ ) (unpaired $t$ test, $p=0.0173, *, p<0.05)$. (D) Quantitative comparison of the immunohistochemical histoscore of LAMTOR5 among grade I $(n=53)$, grade II $(n=121)$ and grade III $(n=36)$ (ordinary one-way ANOVA, $p>0.05)$. (E) Quantitative comparison of the immunohistochemical histoscore of LAMTOR5 among T1 $(n=32)$, T2 $(n=113)$, T3 $(n=44)$ and T4 $(n=21)$. (ordinary one-way ANOVA, $p>0.05)$. (F) Quantitative comparison of the immunohistochemical histoscore of LAMTOR5 between primary HNSCC (SCC, $n=210)$ and recurrence $(n=25)($ unpaired $t$ test, $p>0.05)$. (G) Quantitative comparison of the immunohistochemical histoscore of LAMTOR5 between primary HNSCC $(S C C, n=210$ ) and pre-operation inductive TPF (TPF, $n=20)$. (unpaired $t$ test, $p>0.05$ ).

\section{Expression of LAMTOR5 was notably correlated with p-AktSer473, p-S6Ser235/236, PD-L1, Galectin 9, VISTA, B7-H4, CD68 and CD163 in human primary HNSCC}

We applied immunohistochemistry on human primary HNSCC tissue microarrays and found increased protein level of LAMTOR5, p-Akt ${ }^{\mathrm{Ser} 473}$, p-S6Ser235/236, PD-L1, Galectin 9, VISTA, B7-H4, CD68 and CD163 (Fig. 3. A). Additionally, hierarchical clustering analysis also indicated that the expression

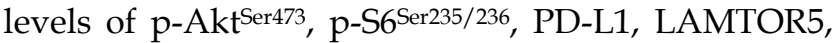
B7-H4, VISTA, Galectin 9, CD68 and CD163 were closely related to each other (Fig. 3. B). Moreover, the 
Spearman rank correlation coefficient test and linear tendency test indicated that the protein expression of LAMTOR5 in HNSCC was significantly correlated with p-Akt ${ }^{\operatorname{Ser} 473}(p<0.001, \mathrm{r}=0.3667), \mathrm{p}-\mathrm{S6}^{\operatorname{Ser} 235 / 236}(p<$ $0.001, \mathrm{r}=0.3711)$, PD-L1 $(p<0.001, \mathrm{r}=0.4251)$, Galectin $9(p<0.001, \mathrm{r}=0.4993)$, VISTA $(p<0.001, \mathrm{r}=$ 0.4456), B7-H4 ( $p<0.001, \mathrm{r}=0.3970), \mathrm{CD} 68(p<0.001, \mathrm{r}$ $=0.3985)$ and CD163 $(p<0.001, r=0.4738)$ (Fig. 4 . A-H).

\section{LAMTOR5 was overexpressed in Pten cKO mice HNSCC and significantly correlated with the expression of VISTA and F4/80}

It has been reported that the deficiency of Pten results in development and progression of HNSCC [28]. In addition, our previous work has indicated that mice with tissue-specific deletion of tumor suppressor gene Pten in epithelia would be more likely to develop HNSCC in a mouse model [29]. Building on these

A

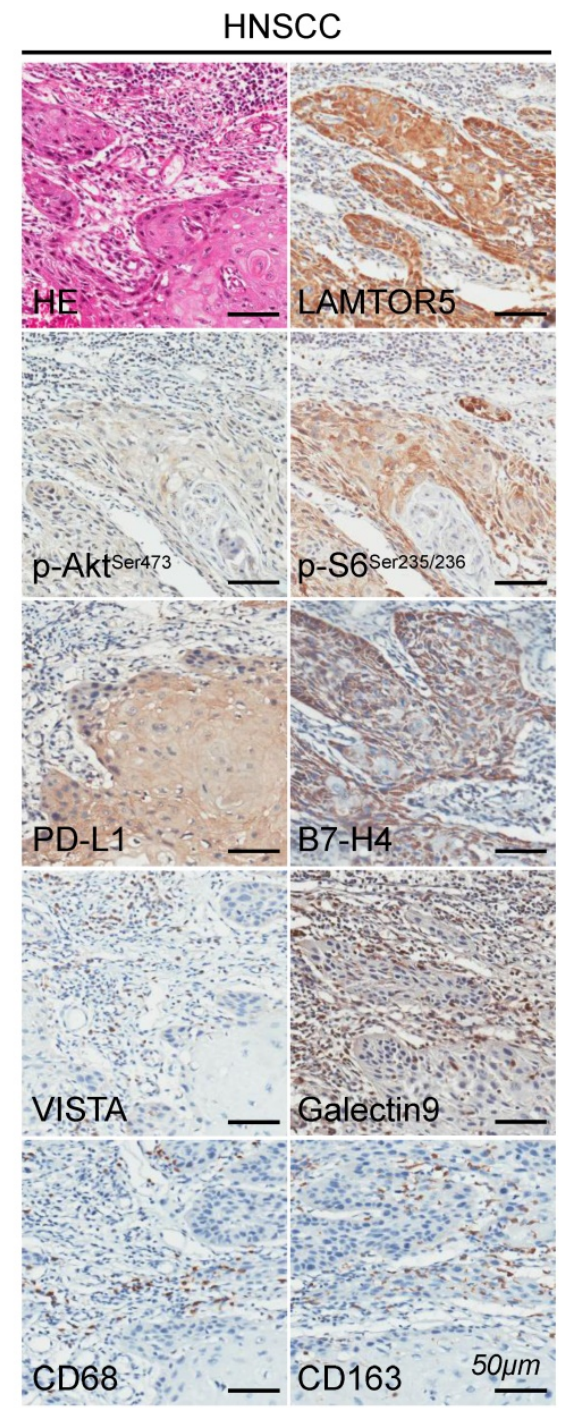

findings, we conducted immunohistochemistry using the LAMTOR5-specific antibody for the purpose of confirming whether LAMTOR5 was overexpressed in Pten cKO mice HNSCC. Indeed, there was an obvious increase of LAMTOR5, as well as F4/80 and VISTA, in the Pten cKO mice HNSCC compared with normal wild-type (WT) mice mucosa (Fig. 5. A). LAMTOR5 was located mostly in the membrane and cytoplasm of the Pten cKO mice cancer cells, consistent with our findings in human HNSCC cells (Fig. 5. A). Meanwhile, Spearman rank correlation coefficient tests and linear tendency tests revealed that the protein expression of LAMTOR5 in Pten cKO mice HNSCC was significantly correlated with VISTA $(p<$ $0.001, r=0.5677)$ and $F 4 / 80(p<0.001, r=0.6790$, Fig. 5. B-C), consistent with the findings in human HNSCC.

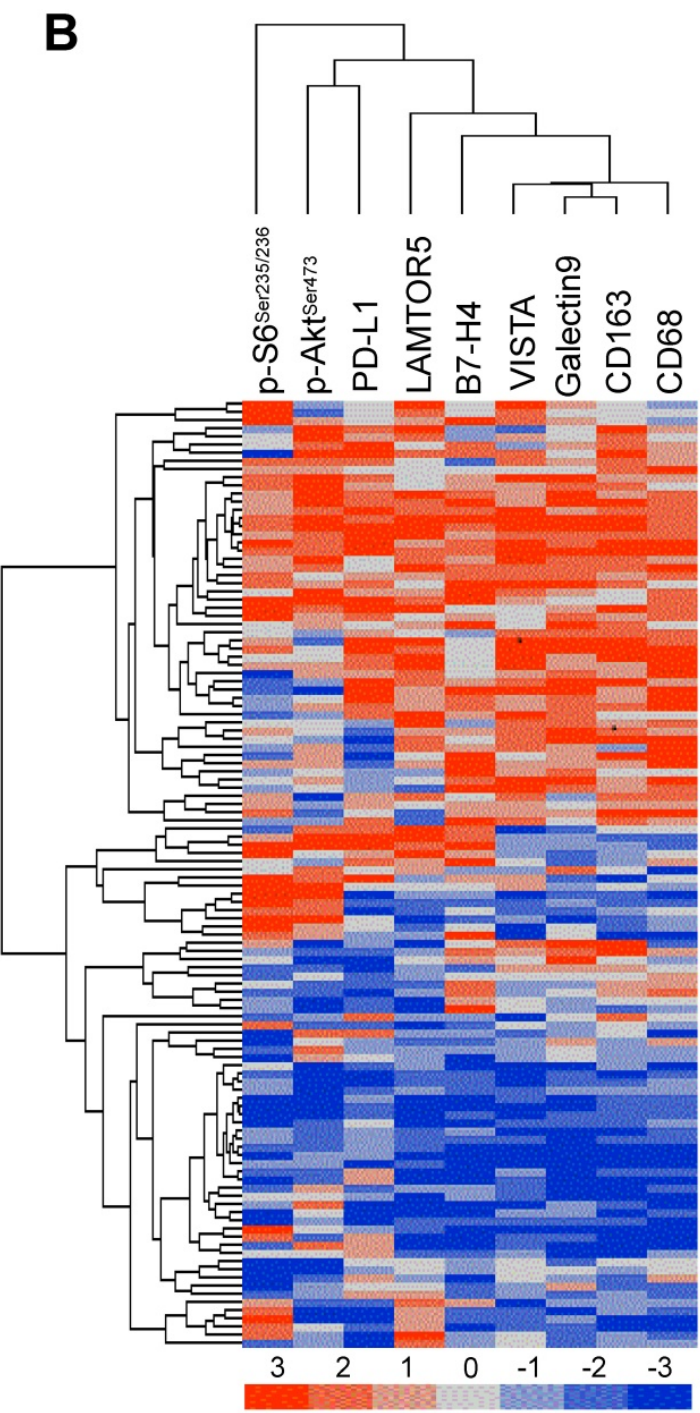

Fig. 3. High expression of LAMTOR5, p-Aktser473, p-S65er235/236, PD-L1, Galectin 9, VISTA, B7-H4, CD68 and CD163 in human primary HNSCC. (A) Representative hematoxylin-eosin (HE) and immunohistochemistry staining of LAMTOR5, p-Akt ${ }^{S e r 473}$, p-S6ser235/236, PD-L1, Galectin 9, VISTA, B7-H4, CD68 and CD163 in human primary HNSCC tissues (Scale bar $=50 \mu \mathrm{m}$ ). (B) Hierarchical clustering shows the affinitive protein expression correlation of LAMTOR5, P-Akt ${ }^{S e r 473}$, P-S6Ser235/236, PD-L1, Galectin 9 , VISTA, B7- H4, CD68 and CD163 in human primary HNSCC. 


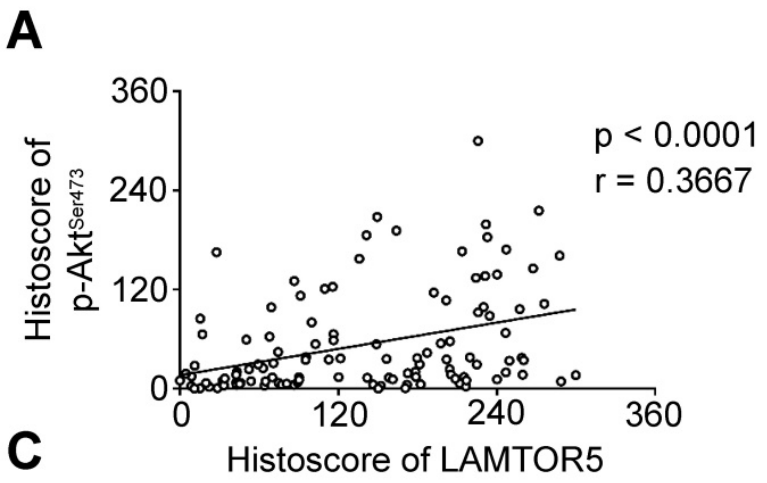

B

C

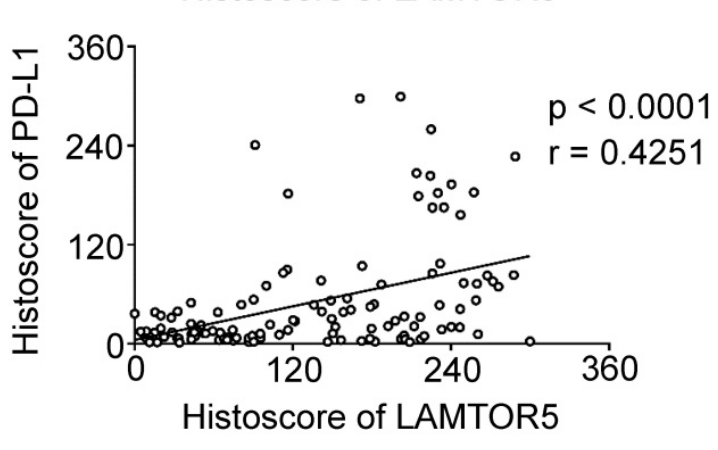

E

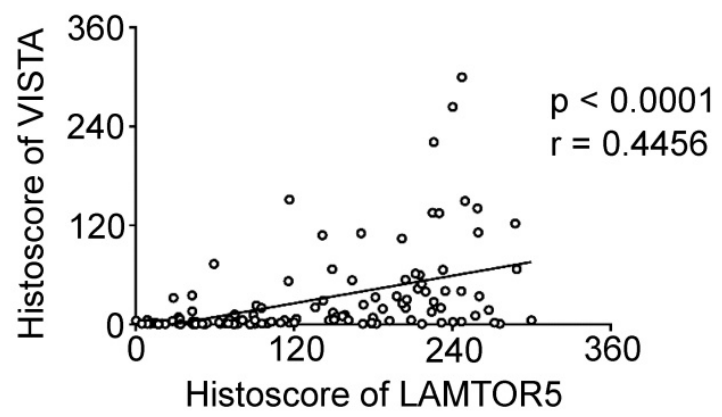

G

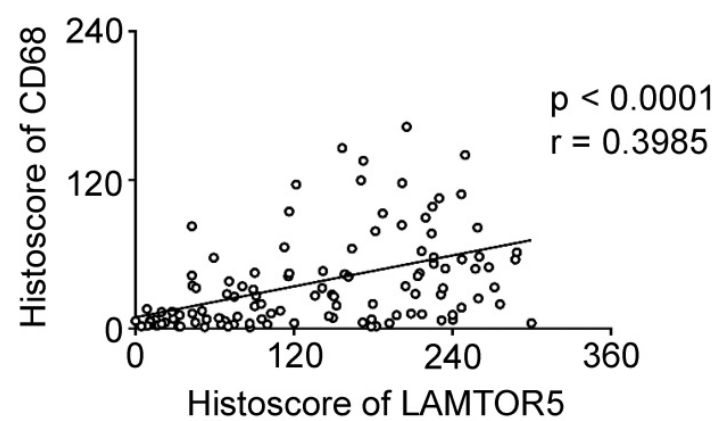

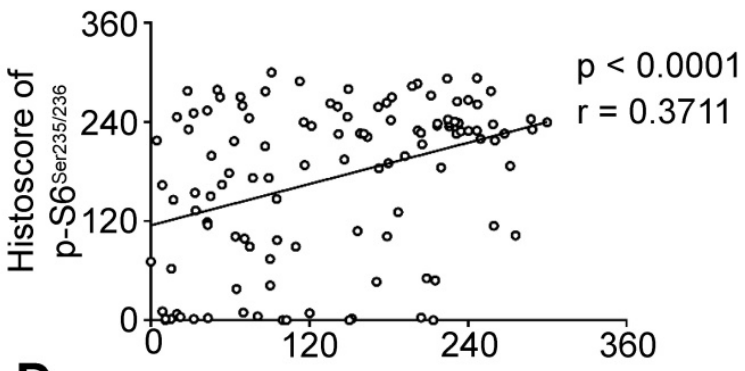

D

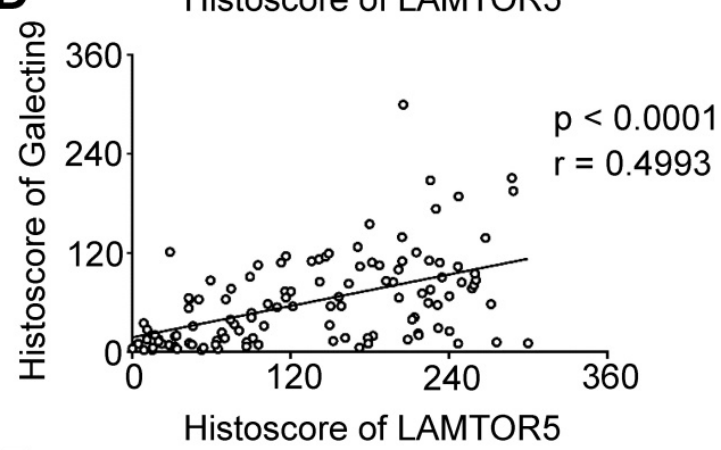

F

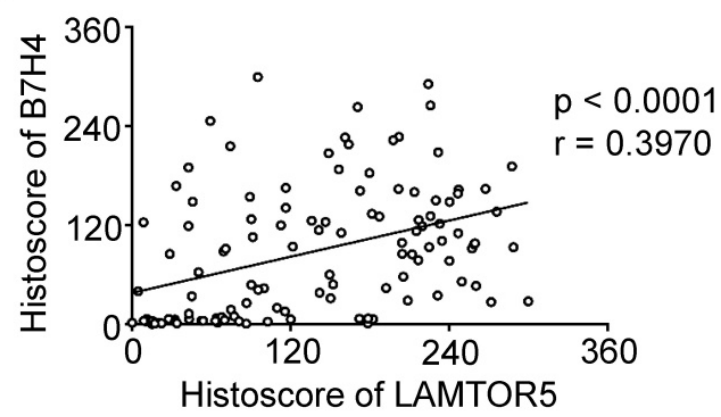

$\mathrm{H}$

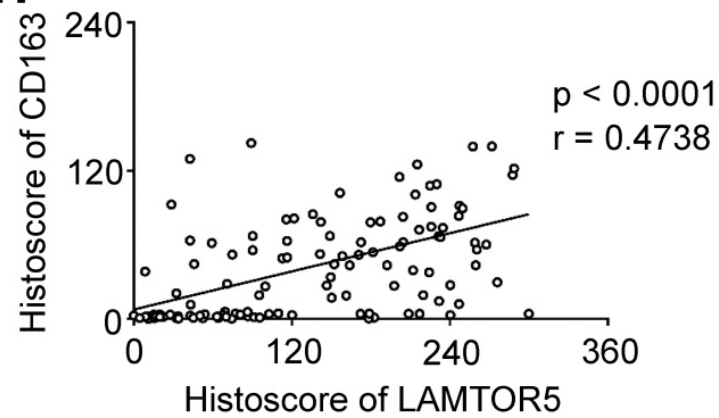

Fig. 4. LAMTOR5 is positively correlated with p-Aktser473, p-S65er235/236, PD-LI, Galectin 9, VISTA, B7- H4, CD68 and CD163 in human primary HNSCC. (A)-(H) Correlation among LAMTOR5 and p-Aktser473, p-S6Ser235/236, PD-LI, Galectin 9, VISTA, B7-H4, CD68 and CD163 in human primary HNSCC.

\section{Discussion}

In our study, it was revealed that overexpression of LAMTOR5 in primary HNSCC was significantly correlated with patients' poor overall survival. LAMTOR5 expression was also significantly associated with lymph node metastasis and lymph node grade. Additionally, LAMTOR5 expression was notably correlated with the expression of $\mathrm{p}-\mathrm{Akt}^{\mathrm{Ser} 473}$,
p-S6 Ser235/236, PD-L1, Galectin 9, VISTA, B7-H4, CD68 and CD163 in human primary HNSCC. Moreover, expression of LAMTOR5 in Pten cKO mouse HNSCC was also positively correlated with VISTA and F4/80 expression. To the best of our knowledge, this is the first study to investigate the expression of LAMTOR5 in human HNSCC as well as Pten cKO mice HNSCC, and to evaluate the clinicopathological and prognostic value of LAMTOR5. 
A

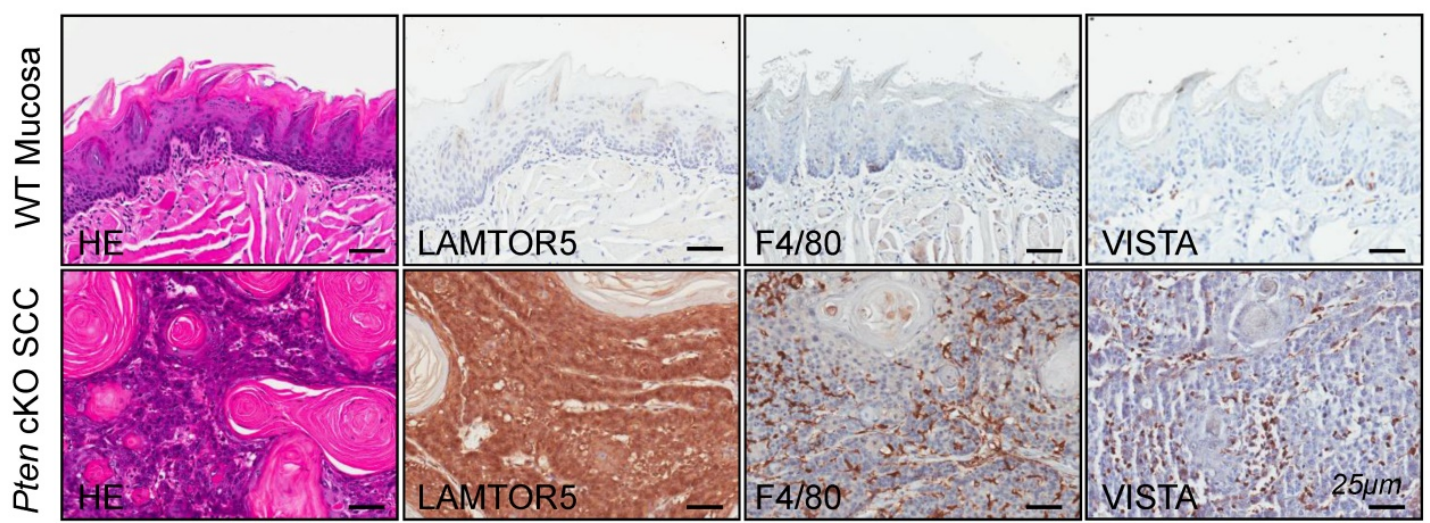

B

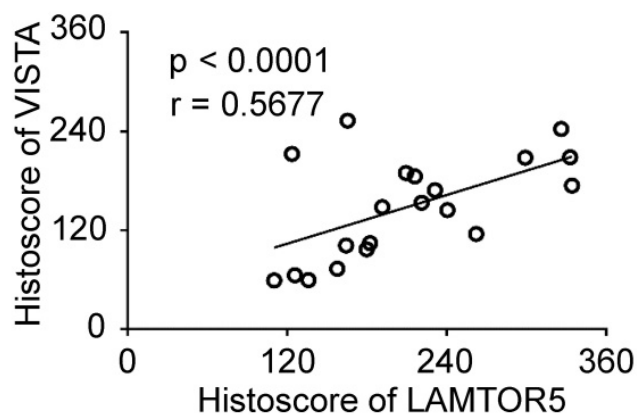

C

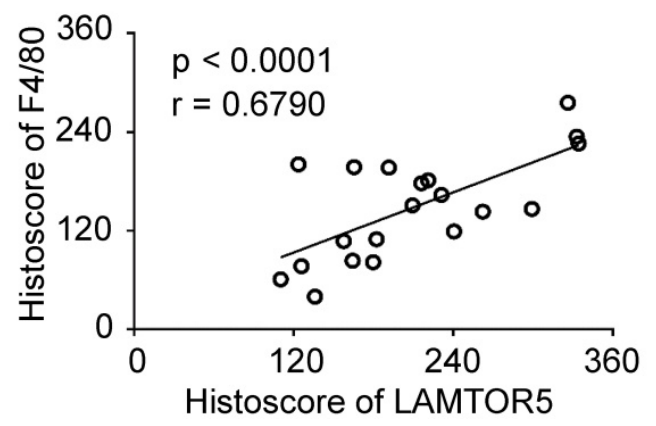

Fig. 5. High expression of LAMTOR5 and the correlation of LAMTOR5 with VISTA and F4/80 in Pten cKO mouse HNSCC. (A) Representative hematoxylin-eosin (HE) and immunohistochemistry of LAMTOR5, VISTA and F4/80 in the wild-type (WT) mice mucosa and the Pten cKO mice HNSCC tissues (Scale bar $=25$ $\mu \mathrm{m})$. (B)-(C) Correlation of LAMTOR5 with VISTA and F4/80 in Pten cKO mice HNSCC.

Acting as an oncoprotein, LAMTOR5 plays complicated roles on cellular level including the promotion of cell growth and proliferation, and the suppression of apoptosis [30-32]. Overexpression of LAMTOR5 has been detected in several malignancies, including esophageal squamous cell carcinoma, cervical cancer, non-small-cell lung cancer and ovarian cancer, and it was worth mentioning that the expression level of LAMTOR5 is also closely associated with the patients' overall survival [9-12]. Our study timely revealed that LAMTOR5 was overexpressed in both human primary HNSCC and Pten CKO mice HNSCC, and the high expression of LAMTOR5 indicated poorer prognosis. The findings above suggested that LAMTOR5 might be both a biomarker for diagnosis and a prognostic indicator in HNSCC.

The PI3K/Akt/mTOR signaling pathway plays an essential role in the development of HNSCC including immune suppression, angiogenesis, and metastasis [33]. Herein we noticed that LAMTOR5 was positively correlated with p-Akt ${ }^{\mathrm{Ser} 473}$ and p-S6Ser235/236. A recent study revealed that by down-regulating Pten, LAMTOR5 activated PI3K/Akt signaling pathway and thereby promoted the migration of breast cancer cells [15]. LAMTOR5 is also crucial to the lysosomal localization of mTORC1 and induces the subsequent activation of mTORC1 [34]. Hence, we speculate that LAMTOR5 might be associated with the metastasis of HNSCC by promoting activation of the PI3K/Akt/mTORC1 signaling pathway.

The inhibitory immune checkpoints suppress immune response toward tumor cells, resulting in tumor immune evasion in HNSCC [20]. With abnormal elevation in HNSCC, B7-H4, VISTA, PD-1 and TIM-3 are inhibitory immune checkpoints, and PD-L1, VISTA and B7-H4 could be prognostic biomarkers in HNSCC [20, 35-37]. We found that LAMTOR5 was significantly correlated with PD-L1 (PD-1 ligand), Galectin 9 (TIM-3 ligand), VISTA and B7-H4 in human primary HNSCC. In the Pten cKO mouse HNSCC model, LAMTOR5 was upregulated and significantly correlated with VISTA. The findings above indicated that LAMTOR5 might play a role in the formation of immunosuppressive tumor microenvironment, and further confirmed that LAMTOR5 might be a poor prognostic indicator.

It has been revealed that tumor-associated macrophages (TAMs) is closely associated with the promotion of tumor-induced angiogenesis, thus leading to poor prognosis in HNSCC [38]. And in breast cancer, TAMs contribute to angiogenesis by activating Akt/GSK-3 $\beta /$ Snail signaling pathway [39]. 
Recent research reported that LAMTOR5 could stimulate tumor-induced angiogenesis via increasing Akt phosphorylation in breast cancer and HepG2 cells $[13,40]$. Our study revealed that LAMTOR5 was significantly correlated with $\mathrm{p}-\mathrm{Akt}^{\mathrm{Ser} 473}$ and human TAM markers (CD68 and CD163). Hence, we suspected that LAMTOR5 might be associated with angiogenesis induced by TAMs via Akt phosphorylation in HNSCC. Moreover, in Pten cKO mouse HNSCC models, LAMTOR5 was also significantly correlated with F4/80, the marker of mouse macrophages [41]. We hypothesized that high expression of LAMTOR5 might be correlate with the infiltration of macrophages into tumor microenvironment. And future functional experiment should be taken to elucidate the relationship of LAMTOR5 expression and macrophages infiltration in HNSCC.

In conclusion, the present study revealed high expression of LAMTOR5 might be a poor prognostic indicator in HNSCC. Taken a step further, LAMTOR5 might be a target for the therapy of HNSCC. The correlation with $\mathrm{p}$-Akt ${ }^{\mathrm{Ser} 473}$, p-S6 $6^{\mathrm{Ser} 235 / 236}$, PD-L1, Galectin 9, VISTA, B7-H4, CD68 and CD163 presented us the probable connections between LAMTOR5 and $\mathrm{PI} 3 \mathrm{~K} / \mathrm{AKT} / \mathrm{mTOR}$ pathway, immune checkpoints and TAMs, suggesting the underlying mechanism of LAMTOR5 including immunosuppression and pro-angiogenesis. However, the correlation analysis of proteins expression was not enough to illustrate the hypothesis. Therefore future functional study of LAMTOR5 in HNSCC is also warranted.

\section{Supplementary Material}

Supplementary figure.

http://www.medsci.org/v16p0783s1.pdf

\section{Acknowledgements}

The present research was supported by the National Nature Science Foundation of China 81874131, 81672668 and 81672667. Zhi-Jun Sun was supported by Fundamental Research Funds for the Central Universities of China 2042017kf0171 (Outstanding Young Scholars) and Hubei Province Nature Science Funds for Distinguished Young Scholar 2017CFA062. We thank for all the patients participating in our research.

\section{Competing Interests}

The authors have declared that no competing interest exists.

\section{References}

1. Saleh K, Eid R, Haddad FG, Khalife-Saleh N, Kourie HR. New developments in the management of head and neck cancer - impact of pembrolizumab. Therapeutics and clinical risk management. 2018; 14: 295-303.
2. Pandey M, Kannepali KK, Dixit R, Kumar M. Effect of neoadjuvant chemotherapy and its correlation with HPV status, EGFR, Her-2-neu, and GADD45 expression in oral squamous cell carcinoma. World journal of surgical oncology. 2018; 16: 20.

3. Goto RN, Sobral LM, Sousa LO, Garcia CB, Lopes NP, Marin-Prida J, et al. Anti-cancer activity of a new dihydropyridine derivative, VdiE-2N, in head and neck squamous cell carcinoma. European journal of pharmacology. 2018; 819: 198-206

4. Leemans CR, Braakhuis BJ, Brakenhoff RH. The molecular biology of head and neck cancer. Nature reviews Cancer. 2011; 11: 9-22.

5. Guan GF, Zhang DJ, Wen LJ, Xin D, Liu Y, Yu DJ, et al. Overexpression of lncRNA H19/miR-675 promotes tumorigenesis in head and neck squamous cell carcinoma. International journal of medical sciences. 2016; 13: 914-22.

6. Melegari M, Scaglioni PP, Wands JR. Cloning and characterization of a novel hepatitis $\mathrm{B}$ virus $\mathrm{x}$ binding protein that inhibits viral replication. Journal of virology. 1998; 72: 1737-43.

7. Hanahan D, Weinberg RA. The hallmarks of cancer. Cell. 2000; 100: 57-70.

8. Fujii R, Zhu C, Wen Y, Marusawa H, Bailly-Maitre B, Matsuzawa S, et al. HBXIP, cellular target of hepatitis B virus oncoprotein, is a regulator of centrosome dynamics and cytokinesis. Cancer research. 2006; 66: 9099-107.

9. Xia H, Ma L, Li J, Bai H, Wang D. Elevated HBXIP expression is associated with aggressive phenotype and poor prognosis in esophageal squamous cell carcinoma. American journal of cancer research. 2017; 7: 2190-8.

10. Wang Y, Sun J, Li N, Che S, Jin T, Liu S, et al. HBXIP overexpression is correlated with the clinical features and survival outcome of ovarian cancer. Journal of ovarian research. 2017; 10: 26

11. Li N, Wang Y, Che S, Yang Y, Piao J, Liu S, et al. HBXIP over expression as an independent biomarker for cervical cancer. Experimental and molecular pathology. 2017; 102: 133-7

12. Wang Y, Li N, Che S, Jin T, Piao J, Liu S, et al. HBXIP suppression reduces cell proliferation and migration and its overexpression predicts poor prognosis in non-small-cell lung cancer. Tumour biology : the journal of the International Society for Oncodevelopmental Biology and Medicine. 2017; 39: 1010428317709675.

13. Wang F, Fei H, Qi B, Yao S, Chang Z. Overexpression of hepatitis B $\mathrm{x}$-interacting protein in HepG2 cells enhances tumor-induced angiogenesis. Molecular and cellular biochemistry. 2012; 364: 165-71.

14. Li Y, Zhang Z, Zhou X, Li L, Liu Q, Wang Z, et al. The oncoprotein HBXIP enhances migration of breast cancer cells through increasing filopodia formation involving MEKK2/ERK1/2/Capn4 signaling. Cancer letters. 2014; 355: 288-96.

15. Liu S, Li L, Zhang Y, Zhang Y, Zhao Y, You X, et al. The oncoprotein HBXIP uses two pathways to up-regulate S100A4 in promotion of growth and migration of breast cancer cells. The Journal of biological chemistry. 2012; 287: 30228-39.

16. Wang FZ, Fei HR, Lian LH, Wang JM, Qiu YY. Hepatitis B x-interacting protein induces HepG2 cell proliferation through activation of the phosphatidylinositol 3-kinase/Akt pathway. Experimental biology and medicine. 2011; 236: 62-9.

17. Wang Z, Martin D, Molinolo AA, Patel V, Iglesias-Bartolome R, Degese MS, et al. mTOR co-targeting in cetuximab resistance in head and neck cancers harboring PIK3CA and RAS mutations. Journal of the National Cancer Institute. 2014; 106

18. Ferris RL. Immunology and Immunotherapy of Head and Neck Cancer. Journal of clinical oncology : official journal of the American Society of Clinical Oncology. 2015; 33: 3293-304.

19. Gildener-Leapman N, Ferris RL, Bauman JE. Promising systemic immunotherapies in head and neck squamous cell carcinoma. Oral oncology. 2013; 49: 1089-96.

20. Deng WW, Wu L, Sun ZJ. Co-inhibitory immune checkpoints in head and neck squamous cell carcinoma. Oral Dis. 2018; 24: 120-3.

21. Msaouel P, Massarelli E. Immune Checkpoint Therapy in Head and Neck Cancers. Cancer J. 2016; 22: 108-16.

22. World Medical A. World Medical Association Declaration of Helsinki. Ethical principles for medical research involving human subjects. Bull World Health Organ. 2001; 79: 373-4

23. Brierley JD, Gospodarowicz MK, Wittekind C. TNM Classification of Malignant Tumours, 8th Edition. New Jersey, USA: John Wiley and Sons; 2016.

24. Huang CF, Zhang L, Ma SR, Zhao ZL, Wang WM, He KF, et al. Clinical significance of Keap1 and Nrf2 in oral squamous cell carcinoma. PloS one. 2013; 8: e83479.

25. Bian Y, Hall B, Sun ZJ, Molinolo A, Chen W, Gutkind JS, et al. Loss of TGF-beta signaling and PTEN promotes head and neck squamous cell carcinoma through cellular senescence evasion and cancer-related inflammation. Oncogene 2012; 31: 3322-32

26. Saldanha AJ. Java Treeview--extensible visualization of microarray data. Bioinformatics. 2004; 20: 3246-8.

27. Budczies J, Klauschen F, Sinn BV, Gyorffy B, Schmitt WD, Darb-Esfahani S, et al. Cutoff Finder: a comprehensive and straightforward Web application enabling rapid biomarker cutoff optimization. PloS one. 2012; 7: e51862.

28. Squarize $\mathrm{CH}$, Castilho RM, Abrahao AC, Molinolo A, Lingen MW, Gutkind JS. PTEN deficiency contributes to the development and progression of head and neck cancer. Neoplasia. 2013; 15: 461-71. 
29. Sun ZJ, Zhang L, Hall B, Bian Y, Gutkind JS, Kulkarni AB. Chemopreventive and chemotherapeutic actions of mTOR inhibitor in genetically defined head and neck squamous cell carcinoma mouse model. Clinical cancer research : an official journal of the American Association for Cancer Research. 2012; 18: 5304-13.

30. Wang FZ, Sha L, Zhang WY, Wu LY, Qiao L, Li N, et al. Involvement of hepatitis B X-interacting protein (HBXIP) in proliferation regulation of cells. Acta pharmacologica Sinica. 2007; 28: 431-8.

31. Marusawa H, Matsuzawa S, Welsh K, Zou H, Armstrong R, Tamm I, et al. HBXIP functions as a cofactor of survivin in apoptosis suppression. The EMBO journal. 2003; 22: 2729-40.

32. Shi H, Fang R, Li Y, Li L, Zhang W, Wang H, et al. The oncoprotein HBXIP suppresses gluconeogenesis through modulating PCK1 to enhance the growth of hepatoma cells. Cancer letters. 2016; 382: 147-56.

33. Vander Broek R, Mohan S, Eytan DF, Chen Z, Van Waes C. The PI3K/Akt/mTOR axis in head and neck cancer: functions, aberrations, cross-talk, and therapies. Oral Dis. 2015; 21: 815-25.

34. Sancak Y, Bar-Peled L, Zoncu R, Markhard AL, Nada S, Sabatini DM. Ragulator-Rag complex targets mTORC1 to the lysosomal surface and is necessary for its activation by amino acids. Cell. 2010; 141: 290-303.

35. Muller T, Braun M, Dietrich D, Aktekin S, Hoft S, Kristiansen G, et al. PD-L1: a novel prognostic biomarker in head and neck squamous cell carcinoma. Oncotarget. 2017; 8: 52889-900.

36. Wu L, Deng WW, Yu GT, Mao L, Bu LL, Ma SR, et al. B7-H4 expression indicates poor prognosis of oral squamous cell carcinoma. Cancer immunology, immunotherapy : CII. 2016; 65: 1035-45.

37. Wu L, Deng WW, Huang CF, Bu LL, Yu GT, Mao L, et al. Expression of VISTA correlated with immunosuppression and synergized with CD8 to predict survival in human oral squamous cell carcinoma. Cancer immunology, immunotherapy : CII. 2017; 66: 627-36.

38. Liss C, Fekete MJ, Hasina R, Lam CD, Lingen MW. Paracrine angiogenic loop between head-and-neck squamous-cell carcinomas and macrophages. International journal of cancer. 2001; 93: 781-5.

39. Lin L, Chen YS, Yao YD, Chen JQ, Chen JN, Huang SY, et al. CCL18 from tumor-associated macrophages promotes angiogenesis in breast cancer. Oncotarget. 2015; 6: 34758-73.

40. Liu F, You X, Wang Y, Liu Q, Liu Y, Zhang S, et al. The oncoprotein HBXIP enhances angiogenesis and growth of breast cancer through modulating FGF8 and VEGF. Carcinogenesis. 2014; 35: 1144-53.

41. McKnight AJ, Macfarlane AJ, Dri P, Turley L, Willis AC, Gordon S. Molecular cloning of F4/80, a murine macrophage-restricted cell surface glycoprotein with homology to the G-protein-linked transmembrane 7 hormone receptor family. The Journal of biological chemistry. 1996; 271: 486-9. 